\title{
Unbiased Minimum-Variance Filter for State and Fault Estimation of Linear Time-Varying Systems with Unknown Disturbances
}

\author{
Fayçal Ben Hmida, ${ }^{1}$ Karim Khémiri, ${ }^{1}$ José Ragot, $^{2}$ \\ and Moncef Gossa ${ }^{1}$ \\ ${ }^{1}$ Electrical Engineering Department, ESSTT-C3S, 5 Avenue Taha Hussein, BP 56, 1008 Tunis, Tunisia \\ ${ }^{2}$ Electrical Engineering Department, CRAN (CNRS UMR 7039), 2 Avenue de la forêt de Haye, \\ 54516 Vandouvre-les-Nancy Cedex, France
}

Correspondence should be addressed to Fayçal Ben Hmida, faycal.benhmida@esstt.rnu.tn

Received 5 October 2009; Revised 2 January 2010; Accepted 11 January 2010

Academic Editor: J. Rodellar

Copyright (C) 2010 Fayçal Ben Hmida et al. This is an open access article distributed under the Creative Commons Attribution License, which permits unrestricted use, distribution, and reproduction in any medium, provided the original work is properly cited.

\begin{abstract}
This paper presents a new recursive filter to joint fault and state estimation of a linear timevarying discrete systems in the presence of unknown disturbances. The method is based on the assumption that no prior knowledge about the dynamical evolution of the fault and the disturbance is available. As the fault affects both the state and the output, but the disturbance affects only the state system. Initially, we study the particular case when the direct feedthrough matrix of the fault has full rank. In the second case, we propose an extension of the previous case by considering the direct feedthrough matrix of the fault with an arbitrary rank. The resulting filter is optimal in the sense of the unbiased minimum-variance (UMV) criteria. A numerical example is given in order to illustrate the proposed method.
\end{abstract}

\section{Introduction}

This paper is concerned with the problem of joint fault and state estimation of linear timevarying discrete-time stochastic systems in the presence of unknown disturbances. In spite of the presence of the unknown inputs, the robust estimate of the state and the fault enables us to implement a Fault Tolerant Control (FTC). A simple idea consists of using an architecture FTC resting on the compensation of the effect of the fault; see, for example, [1].

Initially, we refer to the unknown input filtering problem largely treated in the literature by two different approaches. The first approach was based on the augmentation of the state vector with an unknown input vector. However, this approach assumes that the model for the dynamical evolution of the unknown inputs is available. When the statistical 
properties of the unknown input are perfectly known, the augmented state Kalman filter (ASKF) is an optimal solution. To reduce computation costs of the ASKF, Friedland [2] developed the two-stage Kalman filter (TSKF). This latter is optimal only for a constant bias. Many authors have extended Friedland's idea to treat the stochastic bias, for example, [3-5]. Recently, Kim et al. [6, 7] have developed an adaptive two-stage Kalman filter (ATSKF). The second approach treats the case when we do not have a prior knowledge about the dynamical evolution of the unknown input. Kitanidis [8] was the first to solve this problem using the linear unbiased minimum-variance (UMV). Darouach et al. [9] extended Kitanidis's filter using a parameterizing technique to have an optimal estimator filter (OEF). Hsieh [10] has developed an equivalent to Kitanidis's filter noted robust two-stage Kalman filter (RTSKF). Later, Hsieh [11] developed an optimal minmum variance filter (OMVF) to solve the performance degradation problem encountered in OEF. Gillijns and Moor [12] have treated the problem of estimating the state in the presence of unknown inputs which affect the system model. They developed a recursive filter which is optimal in the sense of minimumvariance. This filter has been extended by the same authors [13] for joint input and state estimation to linear discrete-time systems with direct feedthrough where the state and the unknown input estimation are interconnected. This filter is called recursive three-step filter (RTSF) and is limited to direct feedthrough matrix with full rank. Recently, Cheng et al. [14] proposed a recursive optimal filter with global optimality in the sense of unbiased minimumvariance over all linear unbiased estimators, but this filter is limited to estimate the state (i.e., no estimate of the unknown input). In [15], the author has extended an RTSF-noted ERTSF, where he solved a general case when the direct feedthrough matrix has an arbitrary rank.

In this paper, we develop a new recursive filter to joint fault and state estimation for linear stochastic, discrete-time, and time-varying systems in the presence of unknown disturbances. We assume that the unknown disturbances affect only the state equation. While, the fault affects both the state and the output equations, as well, we consider that the direct feedthrough matrix has an arbitrary rank [15].

This paper is organized as follows. Section 2 states the problem of interest. Section 3 is dedicated to the design of the proposed filter. In Section 4, the obtained filter is summarized. An illustrative example is presented in Section 5. Finally, in Section 6 we conclude our obtained results.

\section{Statement of the Problem}

Assume the following linear stochastic discrete-time system:

$$
\begin{aligned}
x_{k+1} & =A_{k} x_{k}+B_{k} u_{k}+F_{k}^{x} f_{k}+E_{k}^{x} d_{k}+w_{k}, \\
y_{k} & =H_{k} x_{k}+F_{k}^{y} f_{k}+v_{k}
\end{aligned}
$$

where $x_{k} \in \mathfrak{R}^{n}$ is the state vector, $y_{k} \in \mathfrak{R}^{m}$ is the observation vector, $u_{k} \in \mathfrak{R}^{r}$ is the known control input, $f_{k} \in \mathfrak{R}^{p}$ is the additive fault vector, and $d_{k} \in \mathfrak{R}^{q}$ is the unknown disturbances. $w_{k}$ and $v_{k}$ are uncorrelated white noise sequences of zero-mean and covariance matrices are $Q_{k} \geq 0$ and $R_{k}>0$, respectively. The disturbance $d_{k}$ is assumed to have no stochastic description and must be decoupled. The initial state is uncorrelated with the white noises 
processes $w_{k}$ and $v_{k}$ and $x_{0}$ is a Gaussian random variable with $\varepsilon\left[x_{0}\right]=\widehat{x}_{0}$ and $\varepsilon\left[\left(x_{0}-\widehat{x}_{0}\right)\left(x_{0}-\right.\right.$ $\left.\left.\widehat{x}_{0}\right)^{T}\right]=P_{0}^{x}$ where $\varepsilon[\cdot]$ denotes the expectation operator. The matrices $A_{k}, B_{k}, F_{k}^{x}, E_{k}^{x}, H_{k}$, and $F_{k}^{y}$ are known and have appropriate dimensions. We consider the following assumptions:

(i) $A_{1}:\left(H_{k}, A_{k}\right)$ is observable,

(ii) $A_{2}: n>m \geq p+q$,

(iii) $A_{3}: 0<\operatorname{rank}\left(F_{k}^{y}\right) \leq p$,

(iv) $A_{4}: \operatorname{rank}\left(H_{k} E_{k-1}^{x}\right)=\operatorname{rank}\left(E_{k-1}^{x}\right)=q$.

The objective of this paper is to design an unbiased minimum-variance linear estimator of the state $x_{k}$ and the fault $f_{k}$ without any information concerning the fault $f_{k}$ and the unknown disturbances $d_{k}$. We can consider that the filter has the following form:

$$
\begin{aligned}
\widehat{x}_{k / k-1} & =A_{k-1} \widehat{x}_{k-1}+B_{k-1} u_{k-1}+F_{k-1}^{x} \widehat{f}_{k-1}, \\
\widehat{f}_{k} & =K_{k}^{f}\left(y_{k}-H_{k} \widehat{x}_{k / k-1}\right), \\
\widehat{x}_{k} & =\widehat{x}_{k / k-1}+K_{k}^{x}\left(y_{k}-H_{k} \widehat{x}_{k / k-1}\right),
\end{aligned}
$$

where the gain matrices $K_{k}^{f} \in \mathfrak{R}^{p \times m}$ and $K_{k}^{x} \in \mathfrak{R}^{n \times m}$ are determined to satisfy the following criteria.

\section{Unbiasedness}

The estimator must satisfy

$$
\begin{aligned}
& \varepsilon\left[\tilde{f}_{k}\right]=\varepsilon\left[f_{k}-\widehat{f}_{k}\right]=0, \\
& \varepsilon\left[\tilde{x}_{k}\right]=\varepsilon\left[x_{k}-\widehat{x}_{k}\right]=0 .
\end{aligned}
$$

\section{Minimum-Variance}

The estimator is determined such that

(i) the mean square errors $\varepsilon\left[\tilde{f}_{k} \tilde{f}_{k}^{T}\right]$ is minimized under the constraint (2.5);

(ii) the trace $\left\{P_{k}^{x}=\mathcal{E}\left[\tilde{x}_{k} \tilde{x}_{k}^{T}\right]\right\}$ is minimized under the constraints (2.5) and (2.6).

\section{Filter Design}

In this section, the fault and the state estimation are considered in the presence of the unknown disturbance in two cases with respect to assumption $A_{3}$. Section 3.1 is dedicated to deriving a UMV state and fault estimation filter if matrix $F_{k}^{y}$ has full $\operatorname{rank}\left(\right.$ i.e., $\operatorname{rank}\left(F_{k}^{y}\right)=p$ ). A general case will be solved by an extension of the UMV state and fault estimation filter in Section 3.2. 


\subsection{UMV Fault and State Estimation}

In this subsection, we will study a particular case when the $\operatorname{rank}\left(F_{k}^{y}\right)=p$. The gain matrices

$K_{k}^{f}$ and $K_{k}^{x}$ will be determined as that (2.3) and (2.4) can give an unbiased estimation of $f_{k}$ and $x_{k}$. In the next, the UMV fault and state estimation are solved.

\subsubsection{Unbiased Estimation}

The innovation error has the following form

$$
\tilde{y}_{k}:=y_{k}-H_{k} \widehat{x}_{k / k-1}=F_{k}^{y} f_{k}+H_{k} E_{k-1}^{x} d_{k-1}+e_{k}
$$

where

$$
\begin{aligned}
e_{k} & =H_{k} \tilde{\bar{x}}_{k / k-1}+v_{k}, \\
\tilde{\bar{x}}_{k / k-1} & =A_{k-1} \tilde{x}_{k-1}+F_{k-1}^{x} \tilde{f}_{k-1}+w_{k-1} .
\end{aligned}
$$

The fault estimation error and the state estimation error are, respectively, given by

$$
\begin{aligned}
\tilde{f}_{k}: & =f_{k}-\widehat{f}_{k} \\
& =\left(I-K_{k}^{f} F_{k}^{y}\right) f_{k}-K_{k}^{f} H_{k} E_{k-1}^{x} d_{k-1}-K_{k}^{f} e_{k}, \\
\tilde{x}_{k}: & =x_{k}-\widehat{x}_{k} \\
& =\left(I-K_{k}^{x} H_{k}\right) \tilde{\bar{x}}_{k / k-1}-K_{k}^{x} F_{k}^{y} f_{k}-\left(K_{k}^{x} H_{k} E_{k-1}^{x}-E_{k-1}^{x}\right) d_{k-1}-K_{k}^{x} v_{k} .
\end{aligned}
$$

The estimators $\widehat{x}_{k}$ and $\widehat{f}_{k}$ are unbiased if $K_{k}^{f}$ and $K_{k}^{x}$ satisfy the following constraints:

$$
\begin{aligned}
& K_{k}^{f} G_{k}=\mathcal{F}_{k}, \\
& K_{k}^{x} G_{k}=\Gamma_{k},
\end{aligned}
$$

where $G_{k}=\left[\begin{array}{ll}F_{k}^{y} & H_{k} E_{k-1}^{x}\end{array}\right], \mathscr{F}_{k}=\left[\begin{array}{ll}I_{p} & 0\end{array}\right]$ and $\Gamma_{k}=\left[\begin{array}{ll}0 & E_{k-1}^{x}\end{array}\right]$.

Lemma 3.1. Let $\operatorname{rank}\left(F_{k}^{y}\right)=p$; under the assumptions $A_{2}$ and $A_{4}$, the necessary and sufficient condition so that the estimators (2.3) and (2.4) are unbiased as matrix $G_{k}$ is full column rank, that is,

$$
\operatorname{rank}\left(G_{k}\right)=\operatorname{rank}\left(\begin{array}{ll}
F_{k}^{y} & H_{k} E_{k-1}^{x}
\end{array}\right)=p+q
$$


Proof. Equations (3.6) and (3.7) can be written as

$$
\left[\begin{array}{c}
K_{k}^{f} \\
K_{k}^{x}
\end{array}\right] G_{k}=\left[\begin{array}{l}
\mathscr{F}_{k} \\
\Gamma_{k}
\end{array}\right] .
$$

The necessary and sufficient condition for the existence of the solution to (3.9) is

$$
\operatorname{rank}\left[\begin{array}{c}
\mathscr{F}_{k} \\
\Gamma_{k} \\
G_{k}
\end{array}\right]=\operatorname{rank}\left(G_{k}\right) .
$$

We clarify (3.10), and we obtain

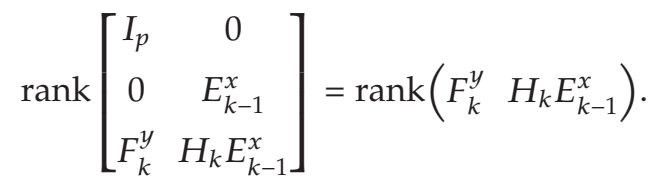

However, the matrix on the left of the equality has a rank equal to $p+q$. According to assumptions $A_{2}, A_{4}$ and $\operatorname{rank}\left(F_{k}^{y}\right)=p$, this can be easily justified by considering that the faults and the unknown disturbances have an independent influences. The condition to satisfy is thus given by (3.8).

\subsubsection{UMV Estimation}

In this subsection, we propose to determine the gain matrices $K_{k}^{f}$ and $K_{k}^{x}$ by satisfying the unbiasedness constraints (2.5) and (2.6).

\section{(a) Fault Estimation}

Equation (3.1) will be written as

$$
\tilde{y}_{k}=G_{k}\left[\begin{array}{c}
f_{k} \\
d_{k-1}
\end{array}\right]+e_{k} .
$$

Since, $e_{k}$ does not have unit variance and $\tilde{y}_{k}$ does not satisfy the assumptions of the GaussMarkov theorem [16], the least-square (LS) solutions do not have a minimum-variance. Nevertheless, the covariance matrix of $e_{k}$ has the following form:

$$
C_{k}=\varepsilon\left[e_{k} e_{k}^{T}\right]=H_{k} \bar{P}_{k / k-1}^{x} H_{k}^{T}+R_{k} \text {, }
$$

where $\bar{P}_{k / k-1}^{x}=\varepsilon\left[\tilde{\bar{x}}_{k / k-1} \widetilde{\bar{x}}_{k / k-1}^{T}\right]$.

For that, $f_{k}$ can be obtained by a weighted least-square (WLS) estimation with a weighting matrix $C_{k}^{-1}$. 
Theorem 3.2. Let $\tilde{\bar{x}}_{k / k-1}$ be unbiased; the matrix $C_{k}$ is positive definite and the matrix $G_{k}$ is full column rank; then to have a UMV fault estimation, the matrix gain $K_{k}^{f}$ is given by

$$
K_{k}^{f *}=\mathcal{F}_{k} G_{k^{\prime}}^{*}
$$

where $G_{k}^{*}=\left(G_{k}^{T} C_{k}^{-1} G_{k}\right)^{-1} G_{k}^{T} C_{k}^{-1}$.

Proof. Under that $C_{k}$ is positive definite and an invertible matrix $S_{k} \in \mathfrak{R}^{m \times m}$ verifes $S_{k} S_{k}^{T}=$ $C_{k}$, so we can rewrite (3.12) as follow.

$$
S_{k}^{-1} \tilde{y}_{k}=S_{k}^{-1} G_{k}\left[\begin{array}{c}
f_{k} \\
d_{k-1}
\end{array}\right]+S_{k}^{-1} e_{k} .
$$

If the matrix $G_{k}$ is full column rank, that is, $\operatorname{rank}\left(G_{k}\right)=p+q$, then the matrix $G_{k}^{T} C_{k}^{-1} G_{k}$ is invertible. Solving (3.15) by an LS estimation is equivalent to solve (3.12) by WLS solution:

$$
\widehat{f}_{k}^{*}=\Psi_{k}\left(G_{k}^{T} C_{k}^{-1} G_{k}\right)^{-1} G_{k}^{T} C_{k}^{-1} \tilde{y}_{k}
$$

In this way, we can consider that $S_{k}^{-1} e_{k}$ has a unit variance and (3.15) can satisfy the assumptions of the Gauss-Markov theorem. Hence, (3.16) is the UMV estimate of $f_{k}$.

In this case, the fault estimation error is rewritten as follows:

$$
\tilde{f}_{k}^{*}=-K_{k}^{f *} e_{k}
$$

Using (3.17), the covariance matrix $P_{k}^{f}$ is given by

$$
P_{k}^{f *}=\varepsilon\left[\tilde{f}_{k}^{*} \tilde{f}_{k}^{* T}\right]=K_{k}^{f *} C_{k} K_{k}^{f * T}=\mp_{k}\left(G_{k}^{T} C_{k}^{-1} G_{k}\right)^{-1} \Psi_{k}^{T}
$$

\section{(b) State Estimation}

In this part, we propose to obtain an unbiased minimum variance state estimator to calculate the gain matrix $K_{k}^{x}$ which will minimize the trace of covariance matrix $P_{k}^{x}$ under the unbiasedness constraint (3.7).

Theorem 3.3. Let $G_{k}^{T} C_{k}^{-1} G_{k}$ be nonsingular; then the state gain matrix $K_{k}^{x}$ is given by

$$
K_{k}^{x *}=\bar{P}_{k / k-1}^{x} H_{k}^{T} C_{k}^{-1}\left(I-G_{k} G_{k}^{*}\right)+\Gamma_{k} G_{k}^{*}
$$

Proof. Considering (3.7) and (3.5), we determine $P_{k}^{x}$ as follows:

$$
\begin{aligned}
P_{k}^{x} & =\left(I-K_{k}^{x} H_{k}\right) \bar{P}_{k / k-1}^{x}\left(I-K_{k}^{x} H_{k}\right)^{T}+K_{k}^{x} R_{k} K_{k}^{x T} \\
& =K_{k}^{x} C_{k} K_{k}^{x T}-2 \bar{P}_{k / k-1}^{x} H_{k}^{T} K_{k}^{x T}+\bar{P}_{k / k-1}^{x} .
\end{aligned}
$$


So, the optimization problem can be solved using Lagrange multipliers:

$$
\operatorname{trace}\left\{K_{k}^{x} C_{k} K_{k}^{x T}-2 \bar{P}_{k / k-1}^{x} H_{k}^{T} K_{k}^{x T}+\bar{P}_{k / k-1}^{x}\right\}-2 \operatorname{trace}\left\{\left(K_{k}^{x} G_{k}-\Gamma_{k}\right) \Lambda_{k}^{T}\right\}
$$

where $\Lambda_{k}$ is the matrix of Lagrange multipliers.

To derive (3.21) with respect to $K_{k}^{x}$, we obtain

$$
C_{k} K_{k}^{* * T}-H_{k} \bar{P}_{k / k-1}^{x}-G_{k} \Lambda_{k}^{T}=0
$$

Equations (3.7) and (3.22) form the linear system of equations:

$$
\left[\begin{array}{cc}
C_{k} & -G_{k} \\
G_{k}^{T} & 0
\end{array}\right]\left[\begin{array}{c}
K_{k}^{x * T} \\
\Lambda_{k}^{T}
\end{array}\right]=\left[\begin{array}{c}
H_{k} \bar{P}_{k / k-1}^{x} \\
\Gamma_{k}^{T}
\end{array}\right]
$$

If $G_{k}^{T} C_{k}^{-1} G_{k}$ is nonsingular, (3.23) will have a unique solution.

\subsubsection{The Filter Time Update}

From (3.3), the prior covariance matrix $\bar{P}_{k / k-1}^{x}=\varepsilon\left[\tilde{\bar{x}}_{k / k-1} \tilde{\bar{x}}_{k / k-1}^{T}\right]$ has the following form:

$$
\bar{P}_{k / k-1}^{x}=\left[\begin{array}{ll}
A_{k-1} & F_{k-1}^{x}
\end{array}\right]\left[\begin{array}{cc}
P_{k-1}^{x *} & P_{k-1}^{x f *} \\
P_{k-1}^{f x *} & P_{k-1}^{f *}
\end{array}\right]\left[\begin{array}{c}
A_{k-1}^{T} \\
F_{k-1}^{x T}
\end{array}\right]+Q_{k-1}
$$

where $P_{k}^{x f *}:=\varepsilon\left[\tilde{x}_{k}^{*} \tilde{f}_{k}^{* T}\right]$ is calculated by using (2.3) and (2.4):

$$
P_{k}^{x f *}=-\left(I-K_{k}^{x *} H_{k}\right) \bar{P}_{k / k-1}^{x} H_{k}^{T} K_{k}^{f * T}+K_{k}^{x *} R_{k} K_{k}^{f * T} .
$$

\subsection{Extended UMV Fault and State Estimation}

In this section, we consider that $0<\operatorname{rank}\left(F_{k}^{y}\right) \leq p$. To solve this interesting problem we will use the proposed approach by Hsieh in (2009) [15]. If we introduce (3.2) and (3.3) into (3.4), then we will be able to write the fault error estimation as follows:

$$
\begin{aligned}
\tilde{f}_{k}:= & \left(I-K_{k}^{f} F_{k}^{y}\right) f_{k}-K_{k}^{f} H_{k} E_{k-1}^{x} d_{k-1}-K_{k}^{f}\left(H_{k} \tilde{\bar{x}}_{k / k-1}+v_{k}\right) \\
= & -K_{k}^{f} H_{k} F_{k-1}^{x} \tilde{f}_{k-1}-K_{k}^{f} H_{k} A_{k-1} \tilde{x}_{k-1}+\left(I-K_{k}^{f} F_{k}^{y}\right) f_{k}-K_{k}^{f} H_{k} E_{k-1}^{x} d_{k-1} \\
& -K_{k}^{f} H_{k} w_{k-1}-K_{k}^{f} v_{k} .
\end{aligned}
$$


Assuming that $\mathcal{E}\left[\tilde{x}_{k-1}\right]=0$ we define the following notations:

$$
\begin{aligned}
\Phi_{k} & =K_{k}^{f} F_{k}^{y}=I_{p}-\Sigma_{k}, \\
G_{k}^{f} & =K_{k}^{f} H_{k} F_{k-1}^{x}, \\
G_{k}^{d} & =K_{k}^{f} H_{k} E_{k-1}^{x},
\end{aligned}
$$

where $\Sigma_{k}=I-\left(F_{k}^{y}\right)^{+} F_{k}^{y}$.

Using the same technique presented in [15], the expectation value of the $\tilde{f}_{k}$ is given by

$$
\begin{aligned}
\varepsilon\left[\tilde{f}_{k}\right]= & \Sigma_{k} f_{k}-G_{k}^{f} \Sigma_{k-1} f_{k-1}+G_{k}^{f}\left(G_{k-1}^{f} \Sigma k-2\right) f_{k-2}+\cdots+(-1)^{k} G_{k}^{f} \times \cdots \times G_{2}^{f}\left(G_{1}^{f} \Sigma_{0}\right) f_{0} \\
& -G_{k}^{d} d_{k-1}+G_{k}^{f} G_{k-1}^{d} d_{k-2}+\cdots+(-1)^{k} G_{k}^{f} \times \cdots \times G_{1}^{f} G_{1}^{d} d_{0} .
\end{aligned}
$$

When we assume that $G_{i}^{f} \Sigma_{i-1}=0$ and $G_{i}^{d}=0$ for $i=1, \ldots, k$, then we obtain

$$
\varepsilon\left[\tilde{f}_{k}\right]=\Sigma_{k} f_{k}
$$

To obtain an unbiased estimation of the fault, the gain matrix $K_{k}^{f}$ should respect the following constraints:

$$
\begin{aligned}
K_{k}^{f} F_{k}^{y} & =\Phi_{k}, \\
K_{k}^{f} H_{k} F_{k-1}^{x} \Sigma_{k-1} & =0, \\
K_{k}^{f} H_{k} E_{k-1}^{x} & =0 .
\end{aligned}
$$

Equation (3.30) can be written as

$$
K_{k}^{f} \bar{G}_{k}=\overline{\boldsymbol{F}}_{k^{\prime}}
$$

where

$$
\begin{aligned}
& \bar{G}_{k}=\left[\begin{array}{lll}
F_{k}^{y} & H_{k} F_{k-1}^{x} \Sigma_{k-1} & H_{k} E_{k-1}^{x}
\end{array}\right], \\
& \overline{\mathscr{F}}_{k}=\left[\begin{array}{lll}
\Phi_{k} & 0 & 0
\end{array}\right] .
\end{aligned}
$$


Using (3.31), we can determine the gain matrix $K_{k}^{f}$ as follows:

$$
K_{k}^{f *}=\overline{\mathscr{F}}_{k} \bar{G}_{k^{\prime}}^{*}
$$

where $\bar{G}_{k}^{*}=\left(\bar{G}_{k}^{T} C_{k}^{-1} \bar{G}_{k}\right)^{+} \bar{G}_{k}^{T} C_{k}^{-1}$ and $X^{+}$denotes the Moore-Penrose pseudoinverse of $X$.

The state estimation error is given by

$$
\begin{aligned}
\tilde{x}_{k}:= & \left(I-K_{k}^{x} H_{k}\right) \tilde{\bar{x}}_{k / k-1}-K_{k}^{x} F_{k}^{y} f_{k}-\left(K_{k}^{x} H_{k} E_{k-1}^{x}-E_{k-1}^{x}\right) d_{k-1}-K_{k}^{x} v_{k} \\
= & \left(I-K_{k}^{x} H_{k}\right) A_{k-1} \tilde{x}_{k-1}+\left(I-K_{k}^{x} H_{k}\right) F_{k-1}^{x} \tilde{f}_{k}-\left(K_{k}^{x} H_{k} E_{k-1}^{x}-E_{k-1}^{x}\right) d_{k-1} \\
& -K_{k}^{x} F_{k}^{y} f_{k}+\left(I-K_{k}^{x} H_{k}\right) w_{k-1}-K_{k}^{x} v_{k} .
\end{aligned}
$$

To have an unbiased estimation of the state, the gain matrix $K_{k}^{x}$ should satisfy the following constraints:

$$
\begin{aligned}
K_{k}^{x} F_{k}^{y} & =0, \\
K_{k}^{x} H_{k} F_{k-1}^{x} \Sigma_{k-1} & =F_{k-1}^{x} \Sigma_{k-1}, \\
K_{k}^{x} H_{k} E_{k-1}^{x} & =E_{k-1}^{x} .
\end{aligned}
$$

From (3.35), we obtain

$$
K_{k}^{x} \bar{G}_{k}=\bar{\Gamma}_{k}
$$

where

$$
\bar{\Gamma}_{k}=\left[\begin{array}{lll}
0 & F_{k-1}^{x} \Sigma_{k-1} & E_{k-1}^{x}
\end{array}\right]
$$

Refering to (3.34), we calculate the error state covariance matrix:

$$
\begin{aligned}
P_{k}^{x} & =\left(I-K_{k}^{x} H_{k}\right) \bar{P}_{k / k-1}^{x}\left(I-K_{k}^{x} H_{k}\right)^{T}+K_{k}^{x} R_{k} K_{k}^{x T} \\
& =K_{k}^{x} C_{k} K_{k}^{x T}-2 \bar{P}_{k / k-1}^{x} H_{k}^{T} K_{k}^{x T}+\bar{P}_{k / k-1}^{x} .
\end{aligned}
$$

The gain matrix $K_{k}^{x}$ is determin by minimizing the trace of the covariance matrix $P_{k}^{x}$ such as (3.36). Using the Kitanidis method [8], we obtain

$$
\left[\begin{array}{cc}
C_{k} & -\bar{G}_{k} \\
\bar{G}_{k}^{T} & 0
\end{array}\right]\left[\begin{array}{c}
K_{k}^{x * T} \\
\Lambda_{k}^{T}
\end{array}\right]=\left[\begin{array}{c}
H_{k} \bar{P}_{k / k-1}^{x} \\
\bar{\Gamma}_{k}^{T}
\end{array}\right],
$$

where $\Lambda_{k}$ is the matrix of Lagrange multipliers. 


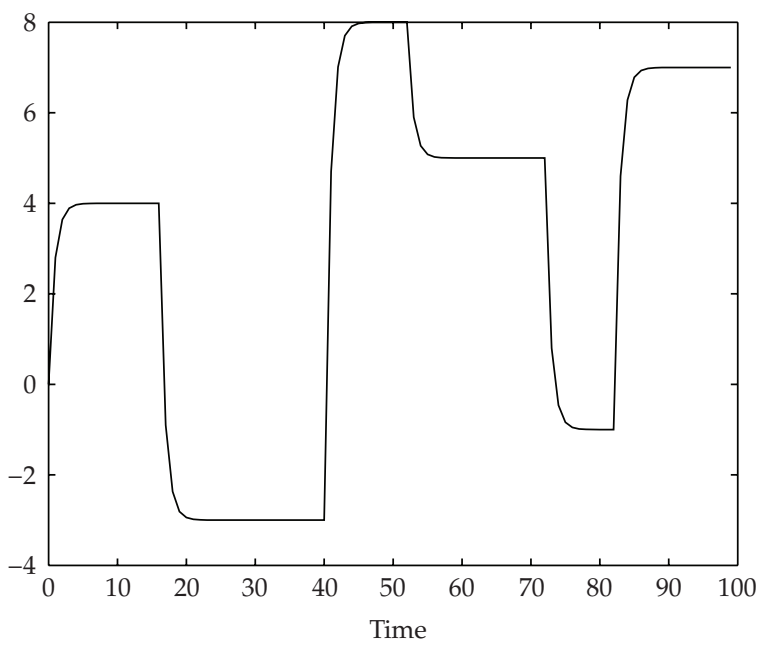

Figure 1: Known input $u_{k}$. is given by

If $\bar{G}_{k}^{T} C_{k}^{-1} \bar{G}_{k}$ is nonsingular, (3.39) will have a unique solution. So, the gain matrix $K_{k}^{x}$

$$
K_{k}^{x *}=\bar{P}_{k / k-1}^{x} H_{k}^{T} C_{k}^{-1}\left(I-\bar{G}_{k} \bar{G}_{k}^{*}\right)+\bar{\Gamma}_{k} \bar{G}_{k}^{*}
$$

The filter time update is the same as that given by (3.24) and (3.25). The obtained filters will be tested by an illustrative example in Section 5 .

\section{Summary of Filter Equations}

We suppose to know the following:

(i) the known input $u_{k}$,

(ii) matrices $A_{k}, B_{k}, H_{k}, F_{k}^{x}, F_{k}^{y}$ and $E_{k^{\prime}}^{x}$

(iii) covariance matrices $Q_{k}^{x}$ and $R_{k}$,

(iv) initial values $\widehat{x}_{0}$ and $P_{0}^{x}$.

We assume that the estimate of the initial state is unbiased and we take the initial covariance matrix $\bar{P}_{0 /-1}^{x}=P_{0}^{x}$. 


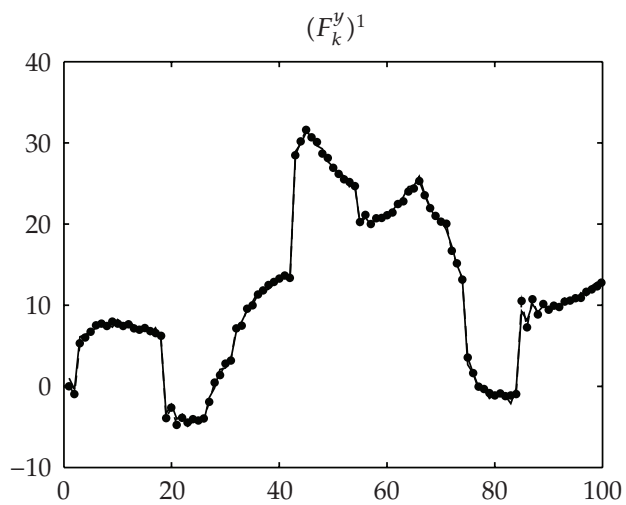

(a)

$\left(F_{k}^{y}\right)^{3}$

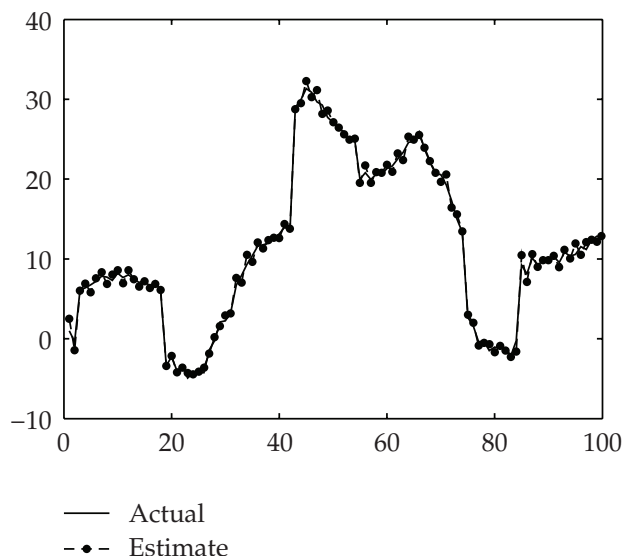

(c)

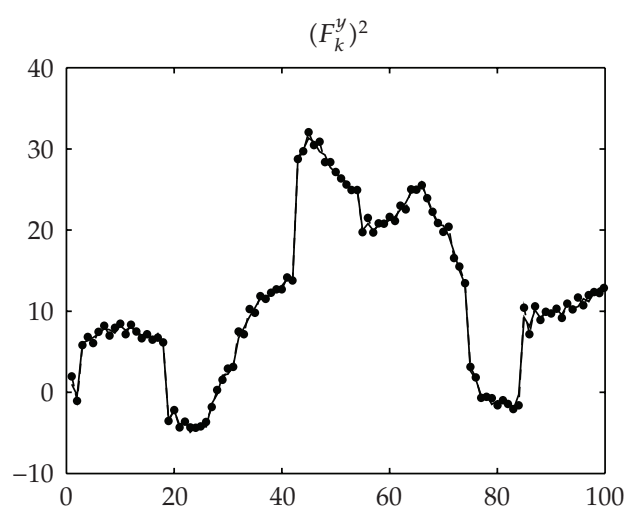

(b)

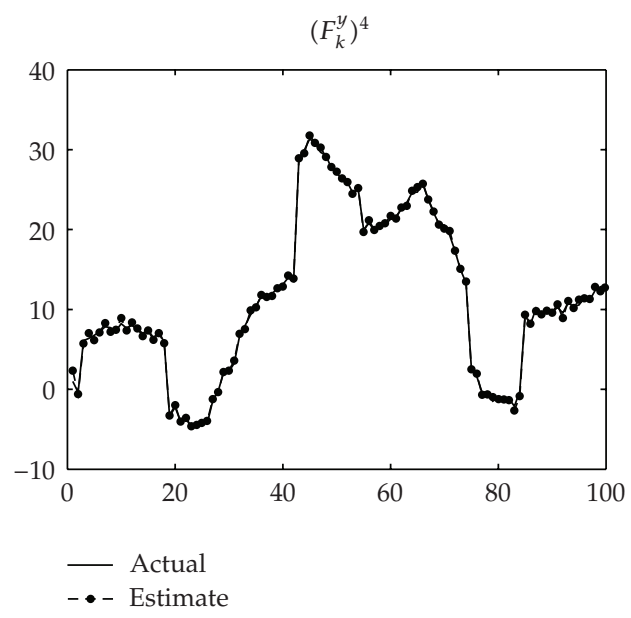

(d)

Figure 2: Actual state $x_{k}^{1}$ and estimated state $\widehat{x}_{k}^{1}$.

Step 1. Estimation of fault is

$$
\begin{aligned}
C_{k} & =H_{k} \bar{P}_{k / k-1}^{x} H_{k}^{T}+R_{k}, \\
\bar{G}_{k} & =\left[\begin{array}{lll}
F_{k}^{y} & H_{k} F_{k-1}^{x} \Sigma_{k-1} & H_{k} E_{k-1}^{x}
\end{array}\right], \\
\bar{\mp}_{k} & =\left[\begin{array}{lll}
\Phi_{k} & 0 & 0
\end{array}\right], \\
\bar{G}_{k}^{*} & =\left(\bar{G}_{k}^{T} C_{k}^{-1} \bar{G}_{k}\right)^{+} \bar{G}_{k}^{T} C_{k}^{-1}, \\
K_{k}^{f} & =\bar{\mp}_{k} \bar{G}_{k}^{*} \\
\widehat{f}_{k} & =K_{k}^{f}\left(y_{k}-H_{k} \widehat{x}_{k / k-1}\right), \\
P_{k}^{f} & =K_{k}^{f} C_{k} K_{k}^{f T} .
\end{aligned}
$$


Step 2. Measurement update is

$$
\begin{aligned}
& \bar{\Gamma}_{k}=\left[\begin{array}{lll}
0 & F_{k-1}^{x} \Sigma_{k-1} & E_{k-1}^{x}
\end{array}\right], \\
& K_{k}^{x}=\bar{P}_{k / k-1}^{x} H_{k}^{T} C_{k}^{-1}\left(I-\bar{G}_{k} \bar{G}_{k}^{*}\right)+\bar{\Gamma}_{k} \bar{G}_{k \prime}^{*} \\
& \widehat{x}_{k}=\widehat{x}_{k / k-1}+K_{k}^{x}\left(y_{k}-H_{k} \widehat{x}_{k / k-1}\right), \\
& P_{k}^{x}=\left(I-K_{k}^{x} H_{k}\right) \bar{P}_{k / k-1}^{x}\left(I-K_{k}^{x} H_{k}\right)^{T}+K_{k}^{x} R_{k} K_{k}^{x T}, \\
& P_{k}^{x f}=-\left(I-K_{k}^{x} H_{k}\right) \bar{P}_{k / k-1}^{x} H_{k}^{T} K_{k}^{f T}+K_{k}^{x} R_{k} K_{k}^{f T} .
\end{aligned}
$$

Step 3. Time update is

$$
\begin{aligned}
& \widehat{x}_{k+1 / k}=A_{k} \widehat{x}_{k}+B_{k} u_{k}+F_{k}^{x} \widehat{f}_{k} \\
& \bar{P}_{k+1 / k}^{x}=\left[\begin{array}{ll}
A_{k} & F_{k}^{x}
\end{array}\right]\left[\begin{array}{cc}
P_{k}^{x} & P_{k}^{x f} \\
P_{k}^{f x} & P_{k}^{f}
\end{array}\right]\left[\begin{array}{c}
A_{k}^{T} \\
F_{k}^{x T}
\end{array}\right]+Q_{k} .
\end{aligned}
$$

Remark 4.1. If $\operatorname{rank}\left(F_{k}^{y}\right)=p$, then we have $\Sigma_{k}=0$ for all $k \geq 0$ and it is easier to use the filter obtained in Section 3.1. In this case, the gain matrices $K_{k}^{f}$ and $K_{k}^{x}$ are given by (3.14) and (3.19), respectively.

Remark 4.2. These remarks give the relationships with the existing literature results.

(i) If $E_{k}^{x}=0$ and $0<\operatorname{rank}\left(F_{k}^{y}\right) \leq p$, the obtained filter is equivalent to ERTSF developed by [15].

(ii) If $E_{k}^{x}=0$ and $\operatorname{rank}\left(F_{k}^{y}\right)=p$, then we have $\Sigma_{k}=0$ for all $k \geq 0$ and the obtained filter is equivalent to RTSF proposed by [13].

(iii) In the case where $F_{k}^{x}=0$ and $F_{k}^{y}=0$, the filter of [8] is obtained.

(iv) In the case where $F_{k}^{x}=0, F_{k}^{y}=0$ and $E_{k}^{x}=0$, we obtain the standard Kalman filter. 


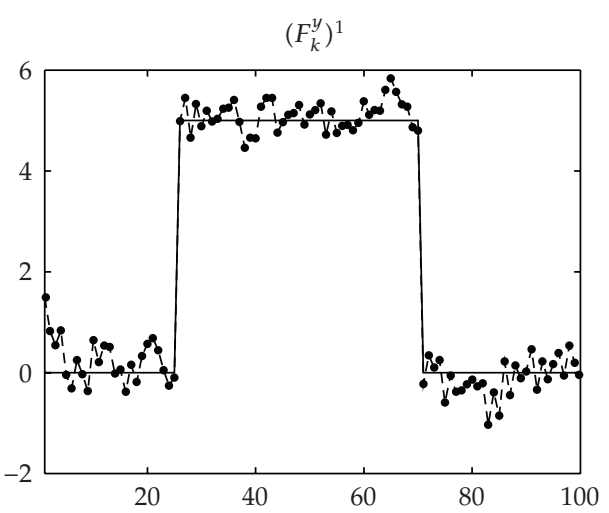

(a)

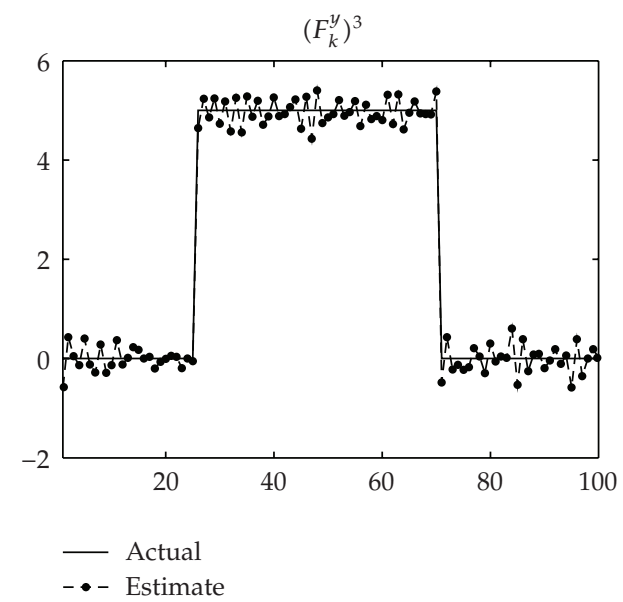

(c)

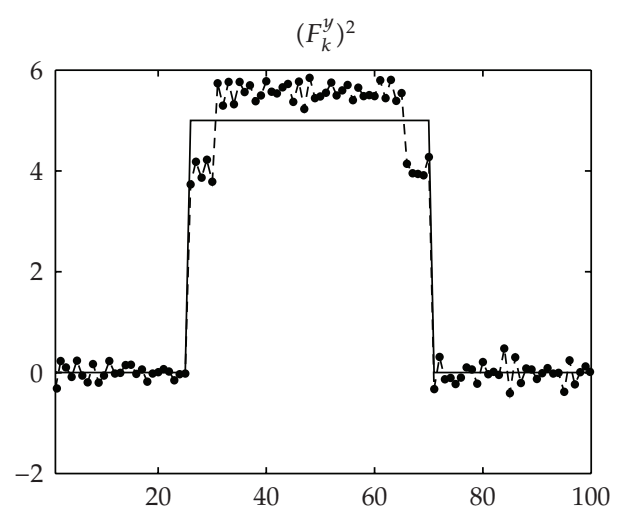

(b)

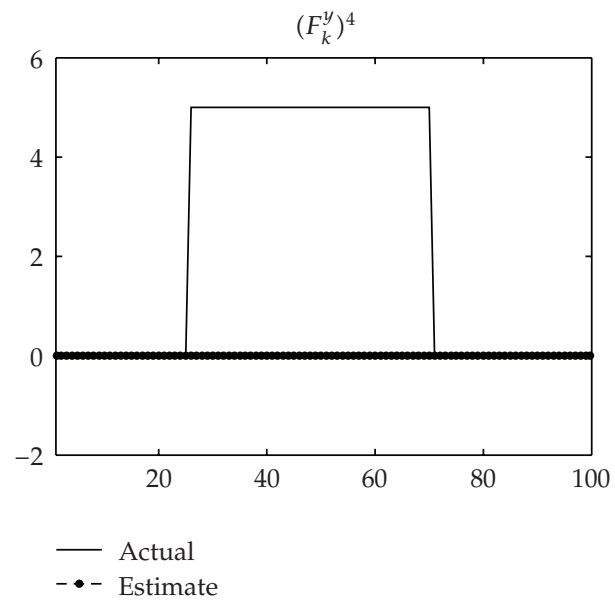

(d)

Figure 3: Actual fault $f_{k}^{1}$ and estimated fault $\widehat{f}_{k}^{1}$.

\section{An Illustrative Example}

To apply our proposed filters we will treat different cases to respect assumption $A_{3}$. The parameters of the system (2.1) are given by

$$
\begin{array}{cc}
x_{k}=\left[\begin{array}{l}
x_{1, k} \\
x_{2, k} \\
x_{3, k}
\end{array}\right], & A_{k}=\left[\begin{array}{lll}
a_{k} & 0.1 & 0.2 \\
0.1 & 0.6 & 0.3 \\
0.5 & 0.1 & 0.25
\end{array}\right], \quad a_{k}=0.4+0.3 \sin (0.2 k), \quad B_{k}=\left[\begin{array}{c}
2 \\
-1.5 \\
0.5
\end{array}\right], \\
F_{k}^{x}=\left[\begin{array}{ll}
0.5 & 0.7 \\
1.5 & 1.1 \\
0.8 & 0.9
\end{array}\right], & E_{k}^{x}=\left[\begin{array}{l}
0 \\
2 \\
1
\end{array}\right], \quad H_{k}=\left[\begin{array}{ccc}
1 & -1 & 0 \\
0 & 1 & 0 \\
0 & -1 & -1
\end{array}\right], \quad Q_{k}=0.1 I_{3 \times 3}, \quad R_{k}=0.01 I_{3 \times 3},
\end{array}
$$




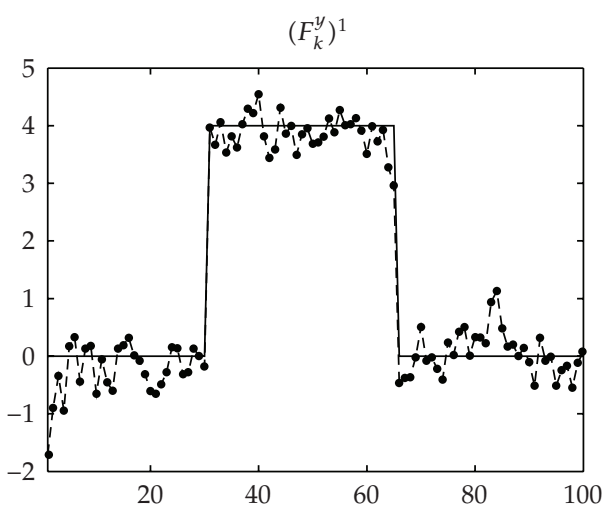

(a)

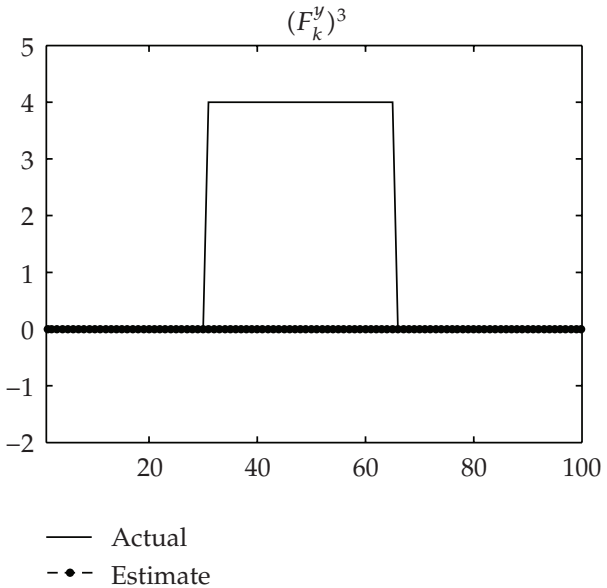

(c)

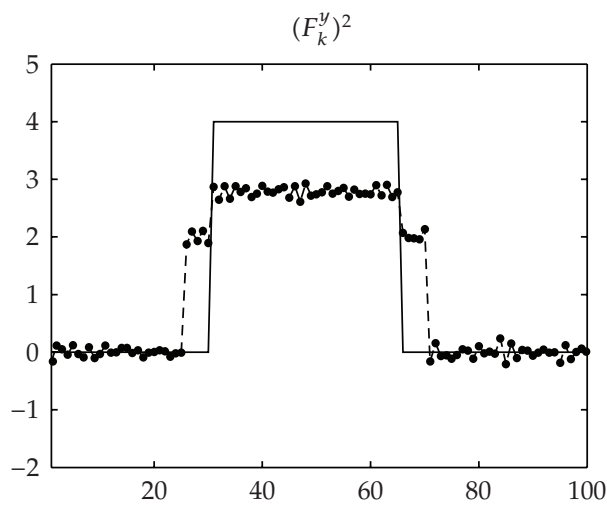

(b)

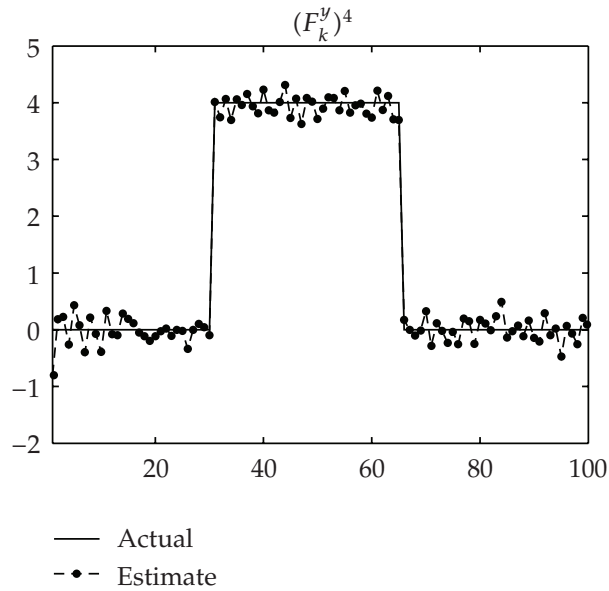

(d)

Figure 4: Actual fault $f_{k}^{2}$ and estimated fault $\widehat{f}_{k}^{2}$.

$$
x_{0}=\left[\begin{array}{c}
1 \\
-2 \\
1
\end{array}\right], \quad \hat{x}_{0}=\left[\begin{array}{l}
0 \\
0 \\
0
\end{array}\right], \quad P_{0}^{x}=I_{3 \times 3} .
$$

In this simulation, four cases of $F_{k}^{y}$ will be considered as follows:

$$
\begin{aligned}
\left(F_{k}^{y}\right)^{1}=\left[\begin{array}{cc}
2 & 1.4 \\
0.6 & 0.3 \\
0.2 & 1.6
\end{array}\right], & \left(F_{k}^{y}\right)^{2}=\left[\begin{array}{cc}
2 & 1 \\
0.6 & 0.3 \\
0.2 & 0.1
\end{array}\right], \\
\left(F_{k}^{y}\right)^{3}=\left[\begin{array}{cc}
2 & 0 \\
0.6 & 0 \\
0.2 & 0
\end{array}\right], & \left(F_{k}^{y}\right)^{4}=\left[\begin{array}{ll}
0 & 1.4 \\
0 & 0.3 \\
0 & 1.6
\end{array}\right] .
\end{aligned}
$$




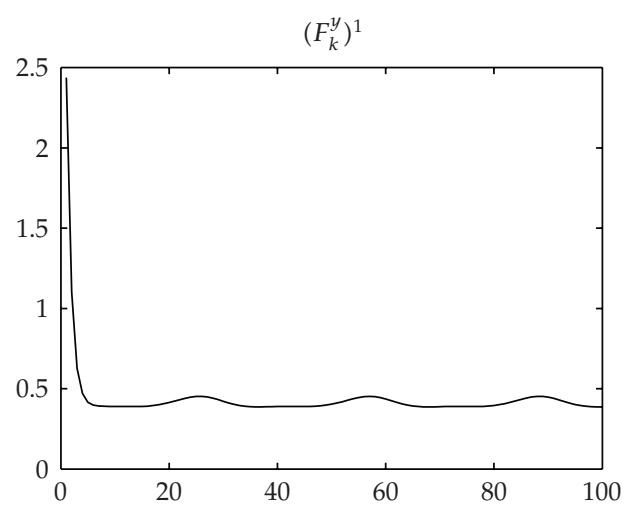

(a)

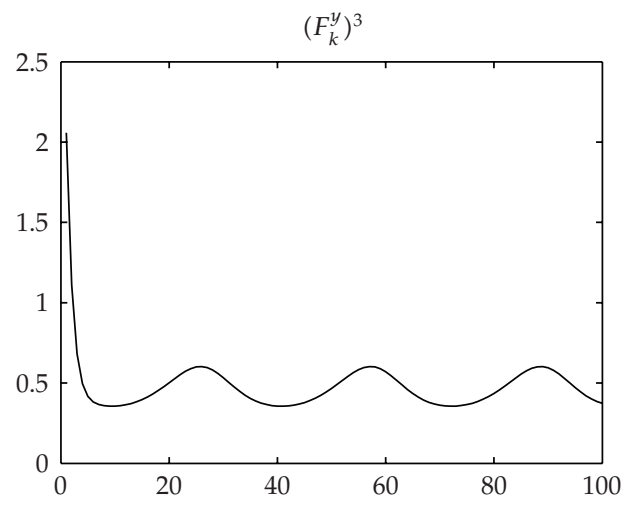

(c)

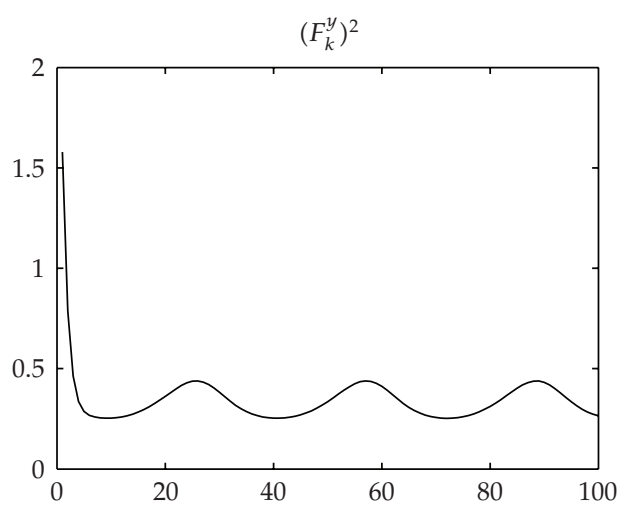

(b)

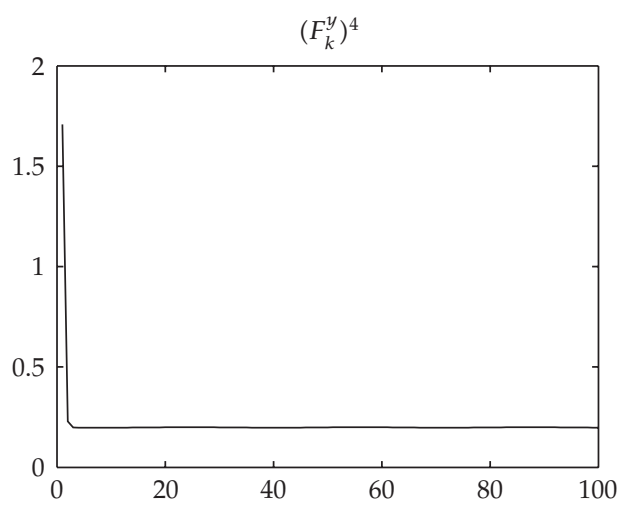

(d)

Figure 5: Trace of the covariance matrix $P_{k}^{x}$.

We assume that the fault and the disturbance are given by

$$
\begin{aligned}
{\left[\begin{array}{l}
f_{1, k} \\
f_{2, k}
\end{array}\right] } & =\left[\begin{array}{l}
5 u_{s}(k-10)-5 u_{s}(k-70) \\
4 u_{s}(k-30)-4 u_{s}(k-65)
\end{array}\right], \\
d_{k} & =4 u_{s}(k-15)-4 u_{s}(k-55),
\end{aligned}
$$

where $u_{s}(k)$ is the unit-step function.

Figure 1 presents the input sequence of the system (2.1). The simulation time is 100 time steps.

In Figure 2, we have plotted the actual and the estimated value of the first element of the state vector $x_{k}=\left[\begin{array}{lll}x_{k}^{1} & x_{k}^{2} & x_{k}^{3}\end{array}\right]^{T}$. Figures 3 and 4 present the actual and the estimated value of the first element and the second element of the fault vector $f_{k}=\left[\begin{array}{lll}f_{k}^{1} & f_{k}^{2}\end{array}\right]^{T}$, respectively. The convergence of the trace of covariance matrices $P_{k}^{x}$ and $P_{k}^{f}$ is shown, respectively, in Figures 5 and 6. 


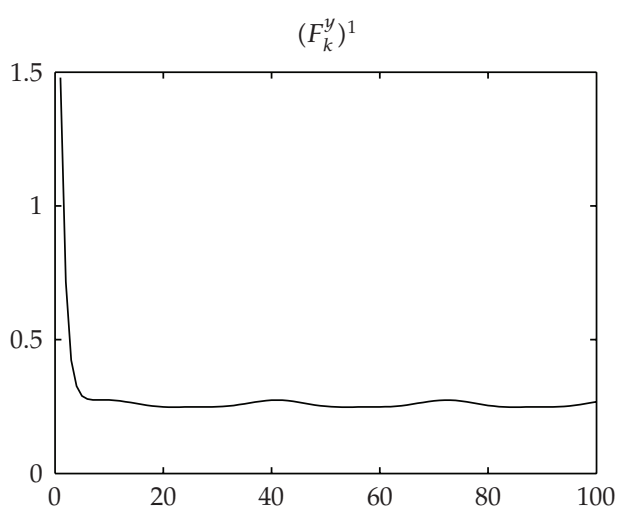

(a)

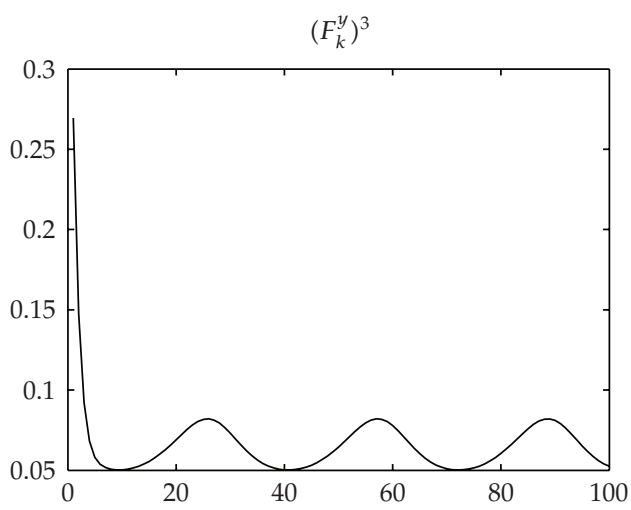

(c)

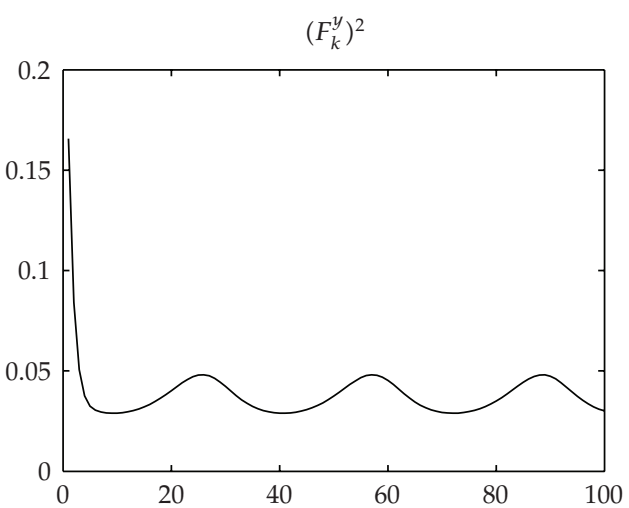

(b)

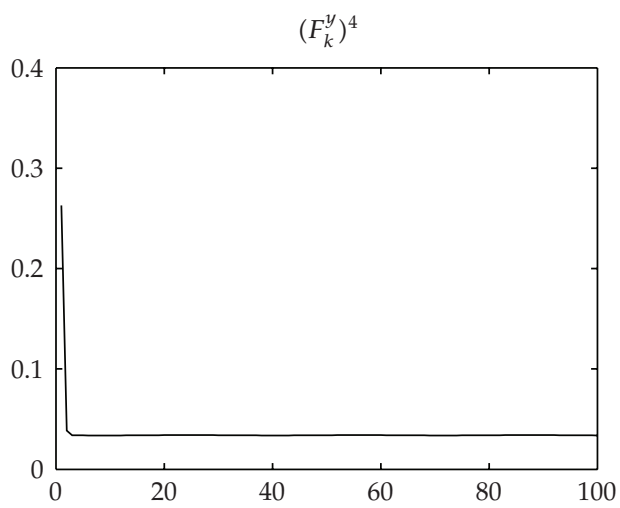

(d)

Figure 6: Trace of the covariance matrix $P_{k}^{f}$.

Table 1: RMSE values.

\begin{tabular}{lccccc}
\hline RMSE & $x_{1, k}$ & $x_{2, k}$ & $x_{3, k}$ & $f_{1, k}$ & $f_{2, k}$ \\
\hline$\left(F_{k}^{y}\right)^{1}$ & 0.4496 & 0.1531 & 0.5114 & 0.3875 & 0.4139 \\
\hline$\left(F_{k}^{y}\right)^{2}$ & 0.5248 & 0.1346 & 0.1863 & 0.4941 & 0.9612 \\
\hline$\left(F_{k}^{y}\right)^{3}$ & 0.6301 & 0.1542 & 0.2129 & 0.2565 & 2.3664 \\
\hline$\left(F_{k}^{y}\right)^{4}$ & 0.3378 & 0.0938 & 0.3647 & 3.3541 & 0.4139 \\
\hline
\end{tabular}

The simulation results in Table 1 show the average root mean square errors (RMSEs) in the estimated states and faults.

According to Figures 2-6 and Table 1, we can conclude that if the matrix $F_{k}^{y}$ has full rank, then we obtain a best estimate of the state and the fault (Figures 2(a), 3(a) and 4(a)). On the other hand, when the matrix $F_{k}^{y}$ has not full rank, it is not possible to obtain a best estimate of the various components of the fault (Figures 3(b), 3(c), 3(d), 4(b), 4(c) and 4(d)), but the state estimation remains acceptable (Figures 2(b), 2(c), and 2(d)). 


\section{Conclusion}

In this paper, the problem of the state and the fault estimation are solved in the case of stochastic linear discrete-time and varying-time systems. A recursive unbiased minimumvariance (UMV) filter is proposed when the direct feedthrough matrix of the fault has an arbitrary rank. The advantages of this filter are especially important in the case when we do not have any priory information about the unknown disturbances and the fault. An application of the proposed filter has been shown by an illustrative example. This recursive filter is able to obtain a robust and unbiased minimum-variance of the state and the fault estimation in spite of the presence of the unknown disturbances.

\section{References}

[1] M. Blanke, M. Kinnaert, J. Lunze, and M. Staroswiecki, Diagnosis and Fault-Tolerant Control, Springer, Berlin, Germany, 2006.

[2] B. Friedland, "Treatment of bias in recursive filtering," IEEE Transactions on Automatic Control, vol. 14, pp. 359-367, 1969.

[3] A. T. Alouani, T. R. Rice, and W. D. A. Blair, "Two-stage filter for state estimation in the presence of dynamical stochastic bias," in Proceedings of the American Control Conference, vol. 2, pp. 1784-1788, Chicago, Ill, USA, 1992.

[4] J. Y. Keller and M. Darouach, "Two-stage Kalman estimator with unknown exogenous inputs," Automatica, vol. 35, no. 2, pp. 339-342, 1999.

[5] C. S. Hsieh and F. C. Chen, "Optimal solution of the two-stage Kalman estimator," IEEE Transactions on Automatic Control, vol. 44, no. 1, pp. 194-199, 1999.

[6] K. H. Kim, J. G. Lee, and C. G. Park, "Adaptive two-stage Kalman filter in the presence of unknown random bias," International Journal of Adaptive Control and Signal Processing, vol. 20, no. 7, pp. 305-319, 2006.

[7] K. H. Kim, J. G. Lee, and C. G. Park, "The stability analysis of the adaptive two-stage Kalman filter," International Journal of Adaptive Control and Signal Processing, vol. 21, no. 10, pp. 856-870, 2007.

[8] P. K. Kitanidis, "Unbiased minimum-variance linear state estimation," Automatica, vol. 23, no. 6, pp. 775-778, 1987.

[9] M. Darouach, M. Zasadzinski, and M. Boutayeb, "Extension of minimum variance estimation for systems with unknown inputs," Automatica, vol. 39, no. 5, pp. 867-876, 2003.

[10] C. S. Hsieh, "Robust two-stage Kalman filters for systems with unknown inputs," IEEE Transactions on Automatic Control, vol. 45, no. 12, pp. 2374-2378, 2000.

[11] C. S. Hsieh, "Optimal minimum-variance filtering for systems with unknown inputs," in Proceedings of the 6th World Congress on Intelligent Control and Automation (WCICA '06), vol. 1, pp. 1870-1874, Dalian, China, 2006.

[12] S. Gillijns and B. Moor, "Unbiased minimum-variance input and state estimation for linear discretetime systems with direct feedthrough," Automatica, vol. 43, no. 5, pp. 934-937, 2007.

[13] S. Gillijns and B. Moor, "Unbiased minimum-variance input and state estimation for linear discretetime systems," Automatica, vol. 43, no. 1, pp. 111-116, 2007.

[14] Y. Cheng, H. Ye, Y. Wang, and D. Zhou, "Unbiased minimum-variance state estimation for linear systems with unknown input," Automatica, vol. 45, no. 2, pp. 485-491, 2009.

[15] C. S. Hsieh, "Extension of unbiased minimum-variance input and state estimation for systems with unknown inputs," Automatica, vol. 45, no. 9, pp. 2149-2153, 2009.

[16] T. Kailath, A. H. Sayed, and B. Hassibi, Linear Estimation, Prentice-Hall, Englewood Cliffs, NJ, USA, 2000. 


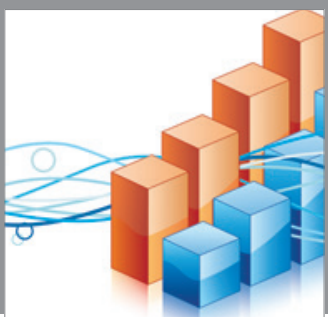

Advances in

Operations Research

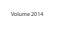

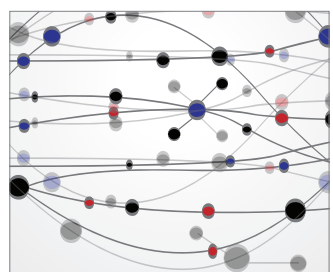

\section{The Scientific} World Journal
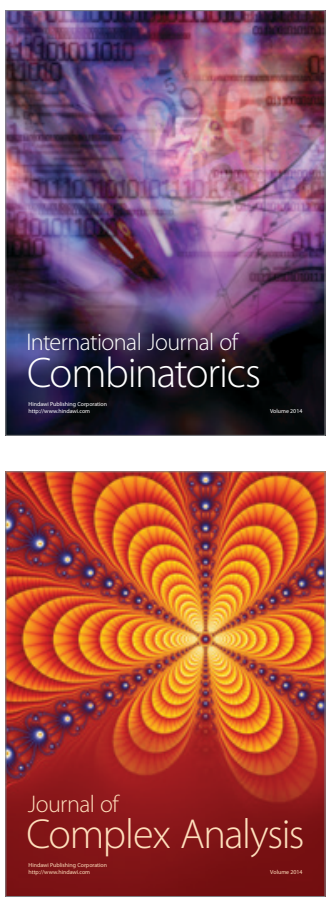

International Journal of

Mathematics and

Mathematical

Sciences
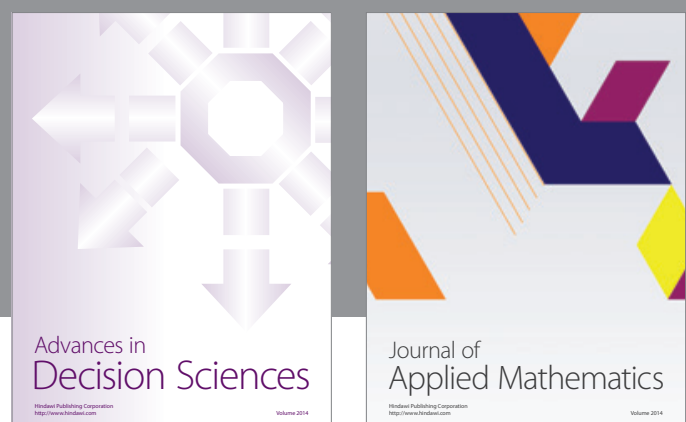

Journal of

Applied Mathematics
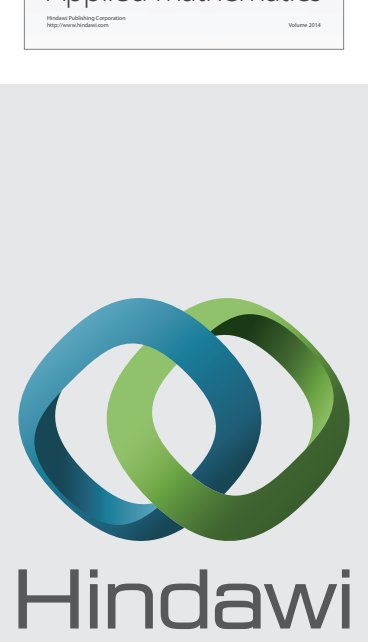

Submit your manuscripts at http://www.hindawi.com
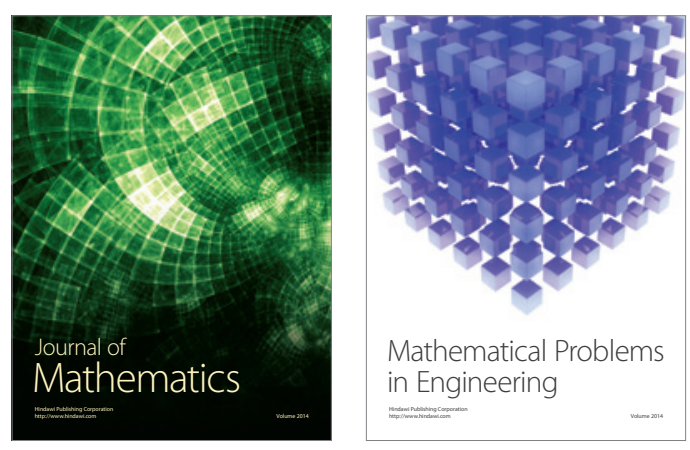

Mathematical Problems in Engineering
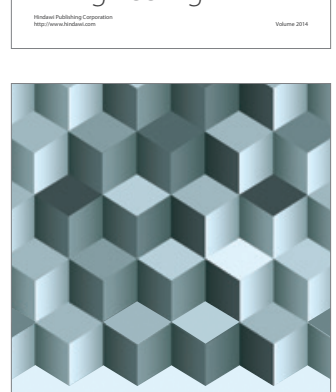

Journal of

Function Spaces
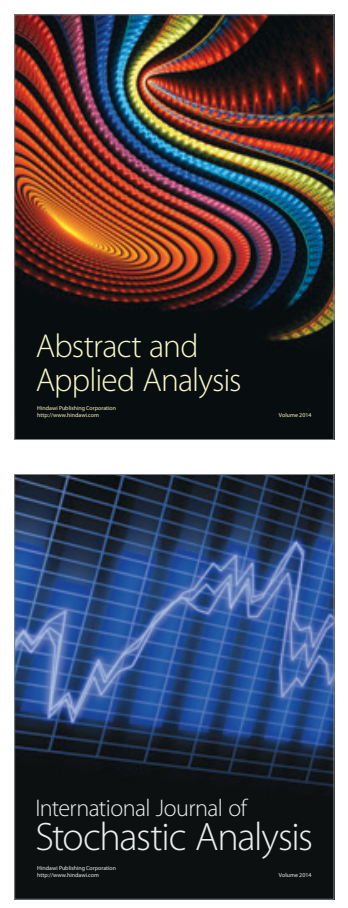

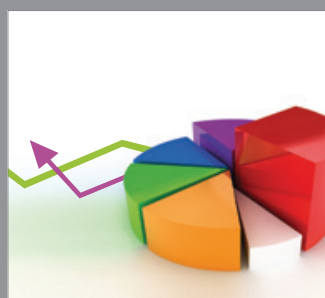

ournal of

Probability and Statistics

Promensencen
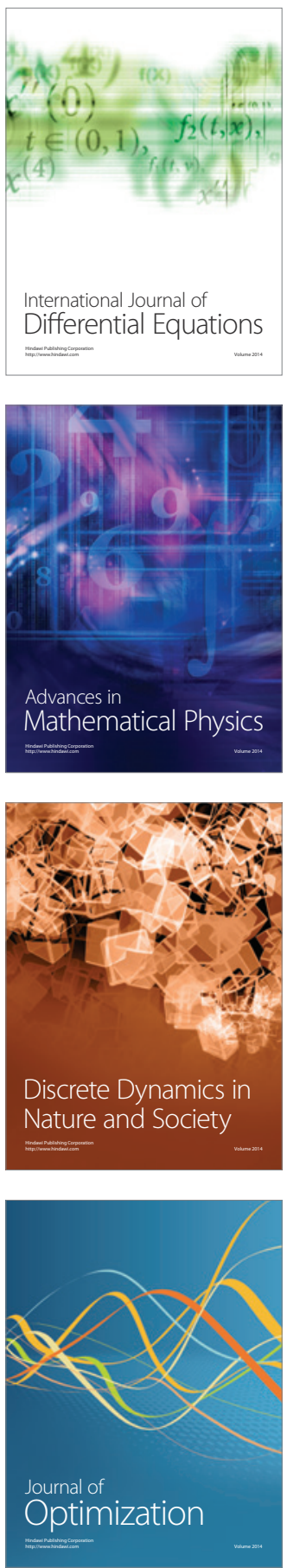